\title{
Double Push Strategy of Knowledge for Product Design Based on Complex Network Theory
}

\author{
Xue-rui Li, Sui-huai Yu, Jian-jie Chu, Deng-kai Chen, and Lin-jian Wu \\ Shaanxi Engineering Laboratory for Industrial Design, Northwestern Polytechnical University, Xian 710072, China \\ Correspondence should be addressed to Xue-rui Li; lxr0829@163.com
}

Received 19 July 2017; Revised 17 October 2017; Accepted 25 October 2017; Published 19 November 2017

Academic Editor: Yong Zhou

Copyright ( 2017 Xue-rui Li et al. This is an open access article distributed under the Creative Commons Attribution License, which permits unrestricted use, distribution, and reproduction in any medium, provided the original work is properly cited.

\begin{abstract}
Reasonable application of design knowledge can help improve the efficiency and quality of product design. Based on complex network theory, this study proposes a double push strategy of knowledge for product design. The proposal introduces the concept of attribute similarity and triangular fuzzy number and uses the theory and method of complex network to build the knowledge network model for product design that contains creative knowledge subnetwork and engineering knowledge subnetwork. This paper is to understand the structure and dynamics of the knowledge network model and to identify and predict knowledge nodes and knowledge groups strongly related to design intent in view of the scale-free network topology analysis theory. We develop a double push strategy of product design knowledge to implement the effective auxiliary function for product design process. Finally, a design case of antalgic pump is presented to demonstrate the practicability and validity of the strategy.
\end{abstract}

\section{Introduction}

Essentially, the product design is a process that designers and engineers use their design knowledge and experience to solve problems on the basis of demand analysis. The process is based on multidomain knowledge creation activities and aimed at building multiple qualities for products, services, and systems that constitute the entire life cycle. However, with the development of information technology, the design process has become extremely complex because of the complexity of object of product. The design process of modern products has evolved into a continuous and complex cognitive process that covers such diverse fields as aesthetics, psychology, behavioral cognition, biology, mechanical engineering, and human engineering. This change requires designers to begin to control the whole product design cycle in the early stage of design process.

The product design process which is the most creative and important stage during the product life cycle determines the final level of product innovation and market success rate. However, a survey [1] shows that designers spend the majority of their time managing design knowledge and less time doing design and analytic work where they can use their specialized expertise to create the greatest value for projects; the results of the survey are shown in Figure 1.

With increasing complexity in design process, it needs optimization team and broad interdisciplinary knowledge to support the creation of designer creative scheme in the design process. It is necessary to rely on accurate intelligent push of knowledge to assist designers in their creative work rather than relying solely on the designer's independent behavior. The double push strategy of knowledge for product design is proposed which aims to assist designers in innovative behavior and design process rather than automation of innovative design.

The remainder of this paper is organized as follows. In Section 2, the related works on knowledge management and push are discussed. Our method for a double push strategy of knowledge is presented in Sections 3, 4, and 5: in Section 3 we firstly construct knowledge model for product design on ontology; in Section 4 we build a knowledge network model for product design; in Section 5 we propose a double oriented push strategy of product design knowledge based complex network theory. Section 6 reports the implementation of the method and shows examples about knowledge push for the product design process of analgesic pump. The conclusion is drawn in Section 7. 


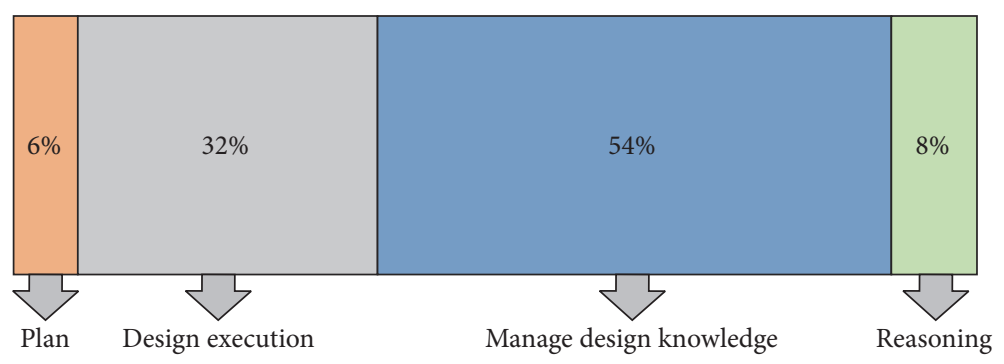

FIgURE 1: The proportion of time spent by designers in design process.

\section{Related Work}

Designers need to acquire a great deal of knowledge during the product design process. Effective management and push of knowledge can help to stimulate innovative thinking, enhance innovation efficiency, and shorten design cycle time [2]. At present, the research of knowledge management theory and method is developing rapidly, and it begins to extend from the economic and management fields to other research and application fields [3]. However, because of involving a number of different areas, including aesthetics, psychology, mechanical processing, and man-machine engineering, design knowledge belongs to complex and diverse unstructured knowledge, which has become a major challenge for knowledge management and push in the design process. Some scholars have begun to study the knowledge management and push method in the design process. In 2011, Park developed a knowledge management system for storing and using the design knowledge acquired in the process of a user-centered design of the next generation information appliances [4]. In 2016, Jansson et al. examined how designers' unique knowledge backgrounds can fuel analogy-based creativity which can support claims from the knowledge-sharing literature for a direct route from knowledge diversity through analogical reasoning to novel idea production [5]. In 2013, Matta et al. proposed a new technique to acquire and represent reusable design knowledge in a design project memory using product lifecycle management platforms [6]. In 2016, Feng et al. proposed an intelligent push method of CNC design knowledge based on latent semantic analysis aiming at the design knowledge demand difference of different designers in CNC design knowledge push process driven by design procedure [7]. In 2015, Liang et al. proposed a dynamic design knowledge push technology based on variable-weight layered spreading activation model to achieve precision and dynamics of product design knowledge push [8]. In 2015, Wang et al. present a new knowledge push technology for complex mechatronic products design based on ontology and variable precision rough set [9]. The diversity and unstructured attributes of design knowledge determine the difficulty of knowledge management and push in product design process. However, the existing methods focus on the representation, modeling, and reuse of design knowledge. Most of these researches are more inclined to develop general knowledge service technology.

Complex network theory mainly uses graph theory and statistical physics to study the topological structure and properties of networks. The discovery of small world networks [10] and scale-free networks [11] has promoted the rapid development of complex networks. At present, there are still few application researches about complex network theory in product design. The theory now mainly focuses on complex mechanical product system, such as network characteristic demonstration $[12,13]$, design process management $[14,15]$, product family design [16], and modular design [17, 18]. Some scholars have started applying complex network theory to knowledge management of mechanical design; for example, Yang et al. proposed a construction method of mechanical design knowledge network based on complex network [19]. However, there is not a yet mature application about complex network theory in product design.

In order to better support product design, this paper proposes a double push method of knowledge for product design based on complex network theory which can achieve more accurate and dynamic knowledge push in product design process.

\section{Product Design Knowledge Modeling}

3.1. Product Design Knowledge. Product design knowledge refers to a set of information accumulated from design theory and work experience to support designers in creative design, structural design, and engineering design. The field of product design knowledge covers aesthetics, psychology, behavioral cognition, biology, mechanical engineering, and ergonomics, which belongs to complex and diverse unstructured knowledge. In product design process, a large number of texts, images, videos, 3D digital models, virtual scenes, and other unstructured information can be used to enrich the designer's thinking space, so as to stimulate new and feasible design concepts. At the same time, materials processing, mechanical engineering, packaging technology, ergonomics, and other knowledge can be used to assist in the completion of the design program. According to the product design process, the design knowledge domain is divided into the creative knowledge domain and the engineering knowledge domain (Figure 2).

(i) Creative Knowledge Domain. The creative knowledge domain stores heuristic abstract knowledge to stimulate creative thinking of designers through visual stimulation. It mainly involves unstructured knowledge such as creative resources, family products, and Pan-Ethnic-Group products $[2]$.

(ii) Engineering Knowledge Domain. The engineering knowledge domain stores the specific knowledge in the different fields of the design and implementation process to assist 


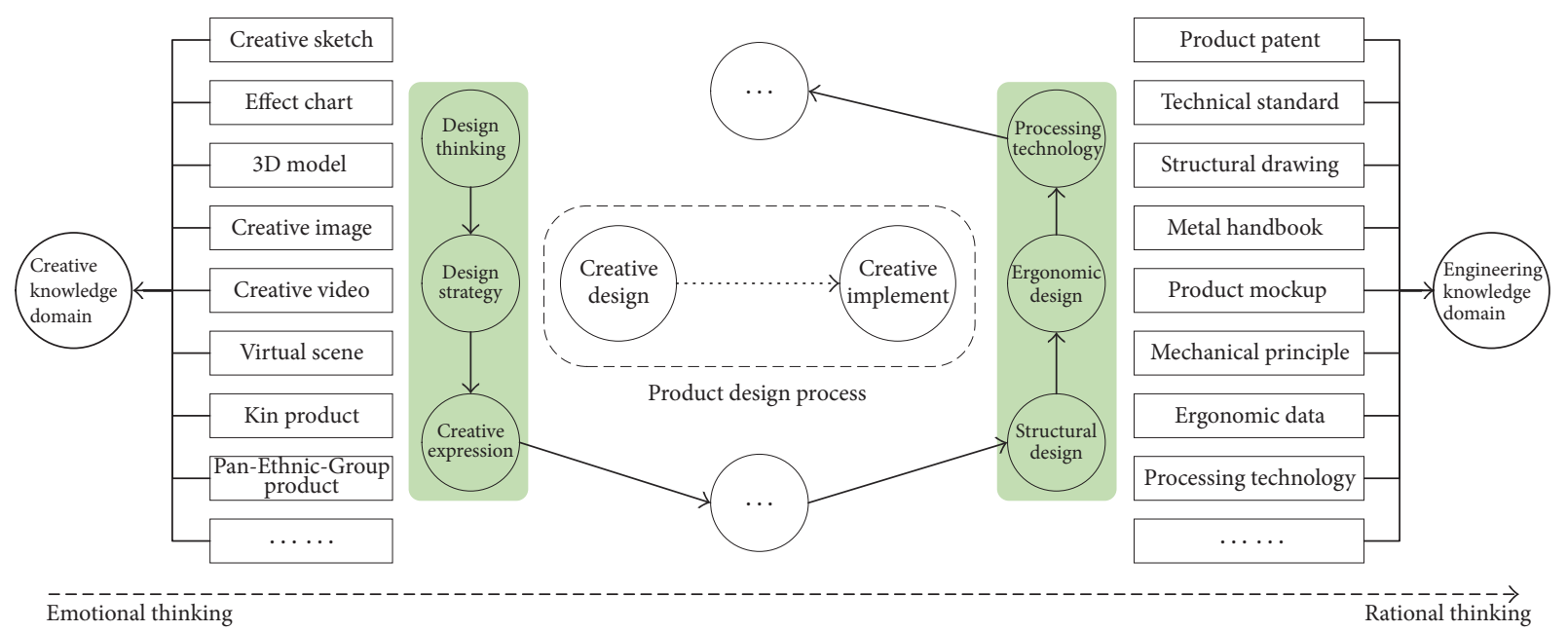

FIGURE 2: Knowledge domain partitioning model based on product design process.

the implementation of the design scheme. It mainly involves structured knowledge such as patents, technical standards, materials, processing technology, and other engineering knowledge.

3.2. Product Design Knowledge Modeling. In order to improve the utilization of design knowledge and actualize the reuse of knowledge to support the innovation design, this paper constructs the product design knowledge model based on ontology and stores the structured knowledge effectively. Because of the difference of knowledge attributes between creative knowledge domain and engineering knowledge domain, this paper classifies product design knowledge during the following research.

3.2.1. Structured Description of the Creative Knowledge Domain. In form, the creative knowledge can be expressed in six-tuple modeling as follows:

$$
\mathrm{KC}=\left(\mathrm{KC}_{\mathrm{id}}, \mathrm{KC}_{t}, \mathrm{KC}_{a}, \mathrm{KC}_{k}, \mathrm{KC}_{i}, \mathrm{KC}_{e}\right) .
$$

The meaning of each symbol is as follows: KC: knowledge in the creative knowledge domain; $\mathrm{KC}_{\mathrm{id}}$ : identification of knowledge; $\mathrm{KC}_{t}$ : the type of knowledge; $\mathrm{KC}_{a}$ : application phase of knowledge; $\mathrm{KC}_{k}$ : Kansei image (in the previous studies, the author has used questionnaires and cluster analysis to extract the 16 Kansei imagery words that best represent the designer's intention in the creative design process [20]); $\mathrm{KC}_{i}$ : the importance of knowledge in its domain; $\mathrm{KC}_{e}$ : the expression of knowledge, including sketch, image, model, and video.

\subsubsection{Structured Description of the Engineering Knowledge} Domain. In form, the engineering knowledge can be expressed in six-tuple modeling as follows:

$$
\mathrm{KE}=\left(\mathrm{KE}_{\mathrm{id}}, \mathrm{KE}_{t}, \mathrm{KE}_{a}, \mathrm{KE}_{f}, \mathrm{KE}_{i}, \mathrm{KE}_{e}\right) .
$$

The meaning of each symbol is as follows: KE: knowledge in the engineering knowledge domain; $\mathrm{KE}_{\mathrm{id}}$ : identification of knowledge; $\mathrm{KE}_{t}$ : the type of knowledge; $\mathrm{KE}_{a}$ : application phase of knowledge; $\mathrm{KE}_{f}$ : the field of knowledge, including material, processing technology, and structure; $\mathrm{KE}_{i}$ : significance degree of knowledge, that is, the importance of knowledge in its domain; $\mathrm{KE}_{e}$ : the expression of knowledge, including data, drawing, manual, and model.

Using the above methods, the product design knowledge base is constructed which includes creative knowledge domain and engineering knowledge domain.

\section{Knowledge Network Model for Product Design}

The knowledge network model for product design (KNmodel) proposed in this paper contains two independent subnetworks: the creative knowledge subnetwork based on the creative knowledge domain and the engineering knowledge subnetwork based on the engineering knowledge domain.

The KN-model can be mathematically described as a graph $G=(N, E)$, where $N=\{1, \ldots, n\}$ is the set of all nodes in the graph and $E \subseteq V \times V$ is the set of edges between pairs of nodes in the network. At the same time, the graph $G=\left\{G_{1}, G_{2}\right\}$, where both $G_{1}$ and $G_{2}$ are subgraphs. The whole network connectivity is completely described by the symmetric adjacency matrix:

$$
A_{i j}=A_{j i}= \begin{cases}1, & (i, j) \in E, \\ 0, & (i, j) \notin E .\end{cases}
$$

Both $A_{i j}$ and $A_{j i}$ are the connection status between $N_{i}$ and $N_{j}: A_{i j}=1$ when link $(i, j)$ exists; that is, there is a relationship between the two nodes; $A_{i j}=0$ otherwise; that is, there is no relationship between the two nodes.

4.1. Fuzzy Importance Calculation. The type of knowledge and the division of knowledge domain depend heavily on the knowledge system administrators and designers. Some of knowledge is classified by administrators and some designers, and a sample library of knowledge types and knowledge domains is obtained. Thus, the feature words of each knowledge type and each knowledge domain are obtained, and 
the corresponding feature vectors are established according to the feature words. The feature vector $F_{v j}$ of knowledge domain $j$ consists of both the feature word and the weight of the feature word in knowledge domain $j$ which can be modified as

$$
F_{v j}=\left\{\left(t_{1}, w_{j 1}^{f}\right),\left(t_{2}, w_{j 2}^{f}\right), \ldots,\left(t_{n}, w_{j n}^{f}\right)\right\},
$$

where $n$ is the dimension of feature vector and $w_{j n}^{f}$ is the weight of feature word $t_{n}$ in knowledge domain $j$.

Fuzzy set theory is a mathematical theory to effectively analyze and deal with incertitude and incompleteness [21]. Triangular fuzzy number is a concept based on the basic principles of rough set theory, which can handle the vagueness and subjectivity.

Definition 1. Let $a=\left(a^{L}, a^{M}, a^{U}\right)$ be a triangular fuzzy number, where $a^{L}$ stands for the lower bound of value, $a^{M}$ stands for the most probable value, and $a^{U}$ stands for the upper bound of value. Then the membership function can be represented as

$$
\mu_{A}(x)= \begin{cases}\frac{\left(x-a^{L}\right)}{\left(a^{M}-a^{L}\right)}, & x \in\left[a^{L}, a^{M}\right], \\ \frac{\left(x-a^{U}\right)}{\left(a^{M}-a^{U}\right)}, & x \in\left[a^{M}, a^{U}\right], \\ 0, & x \in\left[-\infty, a^{L}\right] \cup\left[a^{U},+\infty\right] .\end{cases}
$$

Definition 2. Suppose $a=\left(a^{L}, a^{M}, a^{U}\right)$ and $b=\left(b^{L}, b^{M}, b^{U}\right)$ are triangular fuzzy numbers. The operation of triangular fuzzy number is derived as

$$
\begin{aligned}
a+b & =\left(a^{L}, a^{M}, a^{U}\right)+\left(b^{L}, b^{M}, b^{U}\right) \\
& =\left(a^{L}+b^{L}, a^{M}+b^{M}, a^{U}+b^{U}\right), \\
k \times a & =\left(k a^{L}, k a^{M}, k a^{U}\right), \quad k \geq 0 .
\end{aligned}
$$

The importance of knowledge is dynamic and fuzzy. In order to facilitate the measurement, we use triangular fuzzy number to characterize fuzzy judgment information. On this basis, this paper proposed a novel evaluation approach to assist in fuzzy importance calculation of knowledge nodes.

Step 1 (establishing fuzzy group evaluation matrix). Suppose there are $s$ experts in the decision-making team and $m$ knowledge domains $G_{i}(i=1,2, \ldots, m)$, and $G_{i}$ contains knowledge nodes $k_{i 1}, k_{i 2}, \ldots, k_{i n}$. Taking the subtask corresponding to $G_{i}$ as the criterion and the knowledge node $k_{i j}(j=1, \ldots, n)$ of $G_{i}$ as the subcriterion, the indirect dominance between each element in the element group $G_{i}$ is compared according to its influence on $k_{i j}$; then the judgment matrix can be constructed:

$$
A^{*}=\left[\begin{array}{cccc}
c_{11}^{*} & c_{12}^{*} & \cdots & c_{1 n}^{*} \\
c_{21}^{*} & c_{21}^{*} & \cdots & c_{2 n}^{*} \\
\vdots & \vdots & \ddots & \vdots \\
c_{n 2}^{*} & c_{n 2}^{*} & \cdots & c_{n n}^{*}
\end{array}\right], \quad(* \in[1, s])
$$

where $c_{i j}^{*}=\left\{c_{i j}^{1}, c_{i j}^{2}, \ldots, c_{i j}^{s}\right\}$ is the triangular fuzzy number corresponding to the evaluation data of $s$ experts in the decision-making team. $c_{i j}^{*}$ can be represented as

$$
\operatorname{TFN}\left(c_{i j}^{*}\right)=\left[c_{i j}^{* L}, c_{i j}^{* M}, c_{i j}^{* U}\right],
$$

where $c_{i j}^{* L}, c_{i j}^{* M}, c_{i j}^{* U}$ are the lower bound, most probable value, and upper bound of $\operatorname{TFN}\left(c_{i j}^{*}\right)$. Therefore,

$$
\operatorname{TFN}\left(c_{i j}^{*}\right)=\left\{\left[c_{i j}^{1 L}, c_{i j}^{1 M}, c_{i j}^{1 U}\right], \ldots,\left[c_{i j}^{s L}, c_{i j}^{s M}, c_{i j}^{s U}\right]\right\} .
$$

Using (6), the average triangular fuzzy number $\operatorname{TFN}\left(c_{i j}\right)$ can be got as

$$
\begin{aligned}
\operatorname{TFN}\left(c_{i j}\right) & =\left[c_{i j}^{L}, c_{i j}^{M}, c_{i j}^{U}\right], \\
c_{i j}^{L} & =\frac{\left(c_{i j}^{1 L}+c_{i j}^{2 L}+\cdots+c_{i j}^{s L}\right)}{s}, \\
c_{i j}^{M} & =\frac{\left(c_{i j}^{1 M}+c_{i j}^{2 M}+\cdots+c_{i j}^{s M}\right)}{s}, \\
c_{i j}^{U} & =\frac{\left(c_{i j}^{1 U}+c_{i j}^{2 U}+\cdots+c_{i j}^{s U}\right)}{s} .
\end{aligned}
$$

Therefore, we get the fuzzy group evaluation matrix $A$,

A

$$
=\left[\begin{array}{cccc}
{\left[c_{11}^{L}, c_{11}^{M}, c_{11}^{U}\right]} & {\left[c_{12}^{L}, c_{12}^{M}, c_{12}^{U}\right]} & \cdots & {\left[c_{1 n}^{L}, c_{1 n}^{M}, c_{1 n}^{U}\right]} \\
{\left[c_{21}^{L}, c_{21}^{M}, c_{21}^{U}\right]} & {\left[c_{22}^{L}, c_{22}^{M}, c_{22}^{U}\right]} & \cdots & {\left[c_{2 n}^{L}, c_{2 n}^{M}, c_{2 n}^{U}\right]} \\
\vdots & \ddots & \vdots \\
{\left[c_{n 1}^{L}, c_{n 1}^{M}, c_{n 1}^{U}\right]} & {\left[c_{n 2}^{L}, c_{n 2}^{M}, c_{n 2}^{U}\right]} & \cdots & {\left[c_{n n}^{L}, c_{n n}^{M}, c_{n n}^{U}\right]}
\end{array}\right] .
$$

Step 2 (calculating initial weights).

$$
D_{i}=\frac{\sum_{j=1}^{n} c_{i j}}{\sum_{i=1}^{n} \sum_{j=1}^{n} c_{i j}}, \quad i=1,2, \ldots, n .
$$

By using (13), the initial weight of each knowledge node can be obtained as

$$
D=\left[\begin{array}{c}
{\left[D_{1}^{L}, D_{1}^{M}, D_{1}^{U}\right]} \\
{\left[D_{2}^{L}, D_{2}^{M}, D_{2}^{U}\right]} \\
\vdots \\
{\left[D_{n}^{L}, D_{n}^{M}, D_{n}^{U}\right]}
\end{array}\right]
$$

The possibility of triangular fuzzy number can be applied to remove fuzziness,

$$
\begin{aligned}
& p\left(D_{i} \geq D_{j}\right)=\mu(d) \\
& = \begin{cases}1, & D_{i} \geq D_{j}, \\
\frac{D_{j}^{L}-D_{i}^{U}}{\left(D_{i}^{M}-D_{i}^{U}\right)+\left(D_{j}^{M}-D_{j}^{L}\right)}, & D_{i} \leq D_{j}, D_{i}^{U} \geq D_{j}^{L}, \\
0, & \text { otherwise. }\end{cases}
\end{aligned}
$$


Therefore, the initial weight of each knowledge node can be got as

$$
R=\left(R_{1}, R_{2}, \ldots, R_{i}, \ldots, R_{n}\right) .
$$

Step 3 (calculating synthetical importance). The importance of design knowledge is related to the weight of the knowledge itself and also to the number of times that the knowledge is retrieved. Therefore, the synthetical importance of design knowledge $\mathrm{KE}_{i}$ can be represented as

$$
\mathrm{KE}_{i}=f\left(R_{i}\right)+g\left(\frac{M_{i}}{\sum_{i=1}^{n} M_{i}}\right),
$$

where $M_{i}$ stands for the number of times that the knowledge node is retrieved and $\sum_{i=1}^{n} M_{i}$ stands for the number of times that all knowledge is retrieved. We introduce retrieval factor $\gamma(0 \leq \gamma \leq 1)$ to transform the separation measures into crisp value:

$$
\mathrm{KE}_{i}=\gamma\left(R_{i}\right)+(1-\gamma)\left(\frac{M_{i}}{\sum_{i=1}^{n} M_{i}}\right) .
$$

Therefore, we can rank all knowledge nodes according to their values of $\mathrm{KE}_{i}$. By setting threshold $\mathrm{KE}_{0}$, we can identify the most important knowledge nodes in a knowledge domain. These knowledge nodes can be used to implement the first directional knowledge push.

4.2. Knowledge Network Modeling. According to the above network construction method, we know that the network is composed of nodes and edges, and the nodes of $\mathrm{KN}$-model are product design knowledge. The key point of network construction is to determine the relationship among nodes, that is, the discovery of the edges. In this paper, we use attribute similarity to discover the relationship between the nodes of the product design knowledge network.

The similarity formula of attribute similarity between knowledge points is established. The concrete calculation method is as follows.

Suppose there are two knowledge nodes $N_{i}$ and $N_{j}$ (containing $M$ attributes), then the attribute similarity for attribute $g$ between $N_{i}$ and $N_{j}$ is

$$
\operatorname{as}_{i j}^{g}=\left\|x_{i}^{g}-\overline{x^{g}}|-| x_{j}^{g}-\overline{x^{g}}\right\| .
$$

The meaning of each symbol is as follows: $x_{i}^{g}$ : the value of $N_{i}$ about the attribute $g ; x_{j}^{g}$ : the value of $N_{j}$ about the attribute $g ; \overline{x^{g}}$ : the average value of all knowledge points about the attribute $g$.

In order to measure the similarity between two knowledge nodes, we need to standardize the attribute similarity.

The specification of attribute similarity between the two knowledge nodes such as $N_{i}$ and $N_{j}$ (containing $M$ attributes) given by the upper segment is

$$
\text { ans }(i, j)=\frac{\sum_{n=1}^{m} \mathrm{as}_{i j}^{n}}{m} \text {. }
$$

Then, ans $(i, j)$ is the attribute similarity between two knowledge nodes; $\mathrm{ans}_{0}$ is the threshold of attribute similarity.
The smaller ans $(i, j)$ is, the more similar two nodes will be. If ans $(i, j) \leq$ ans $_{0}$, it can make clear the relation between the two product design knowledge nodes, then an edge can be created between two nodes. By calculating the attribute similarity between all nodes in $\mathrm{KN}$-model, the relationships between the nodes in $\mathrm{KN}$-model can be determined.

\section{A Double Oriented Push Strategy of Design Knowledge}

5.1. Immunization Strategy in Complex Network. In order to enhance the novelty of product design knowledge and to stimulate innovation, immunization strategy can be used to immunize some of the nodes in the network. The study found that the acquaintance immunization strategy is effective for any broad-scale distributed network [22]. Figure 4 shows an improved acquaintance immunization strategy which is proposed in this paper and the immune process is as follows.

Step 1. Randomly select $X$ nodes from all knowledge nodes in $K N$-model.

Step 2. Determine whether $K_{i}$ is confirmed. If confirmed, proceed to the next decision; if not confirmed, randomly immunize $m$ neighboring nodes.

Step 3 (if $K_{i} \geq K_{\text {cut }}$ ). Immunize node $N_{i}$, and then randomly immunize a neighboring node of $N_{i}$.

Step 4 (if $K_{i}<K_{\text {cut }}$ ). First, randomly immunize $m$ neighboring nodes; second, randomly select $a$ node $\left(N_{j}\right)$ from $m$ nodes; finally, randomly immunize $a$ neighboring node of $N_{i}$.

\subsection{Network Topology Analysis}

5.2.1. Importance Evaluation of Knowledge Nodes. The importance evaluation of knowledge nodes can help designers to extract knowledge nodes that are most closely related to a design intention so as to give priority to push and enhance the efficiency of innovation. The importance of a node is mainly expressed in the centrality of the node, that is, the number of nodes that are related to the node. In this paper, we adopt the concept of degree and degree distribution in complex networks to mine the most central nodes in the knowledge network.

According to the concept of graph theory, the cumulative degree distribution function of $\mathrm{KN}$-model is calculated:

$$
P_{k}=\sum_{k^{\prime}=k}^{\infty} P\left(k^{\prime}\right) .
$$

It can be verified that if the node-degree distribution is a power law of the form $P(k) \sim k^{-r}$ with a constant $r>0$, then

$$
P_{k} \propto \sum_{k^{\prime}=k}^{\infty} k^{\prime-r} \propto k^{-(r-1)} .
$$

It can be proved that the $\mathrm{KN}$-model constructed in Figure 3 is a scale-free network. Most of the knowledge 


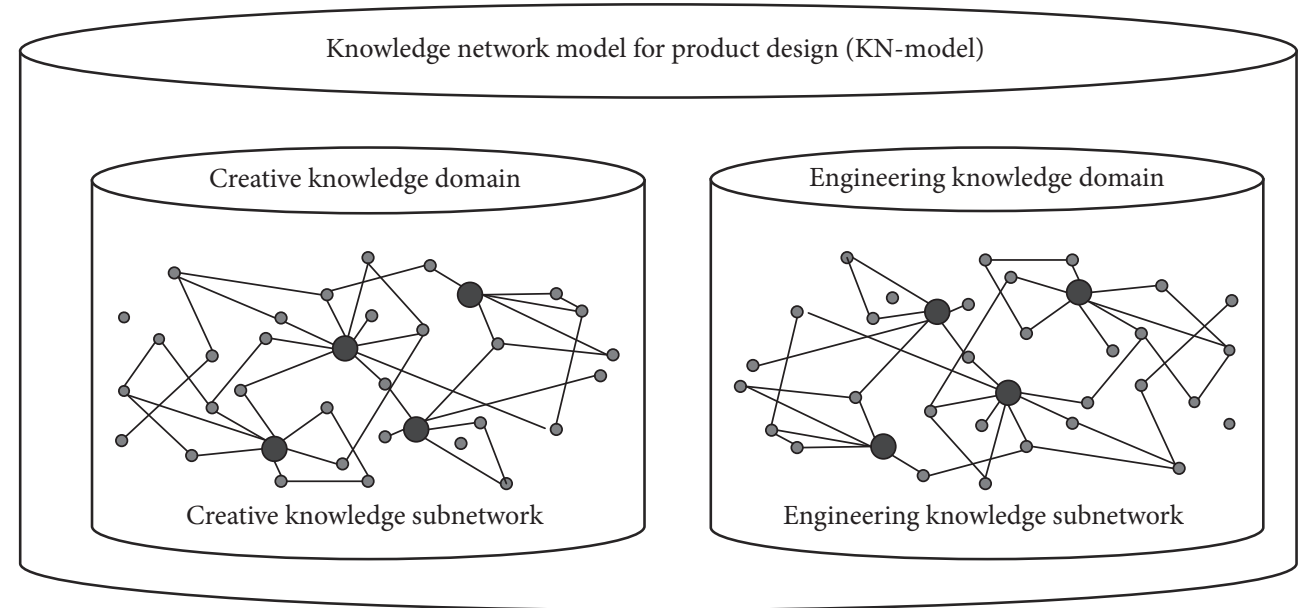

FIGURE 3: The knowledge network model for product design (KN-model).

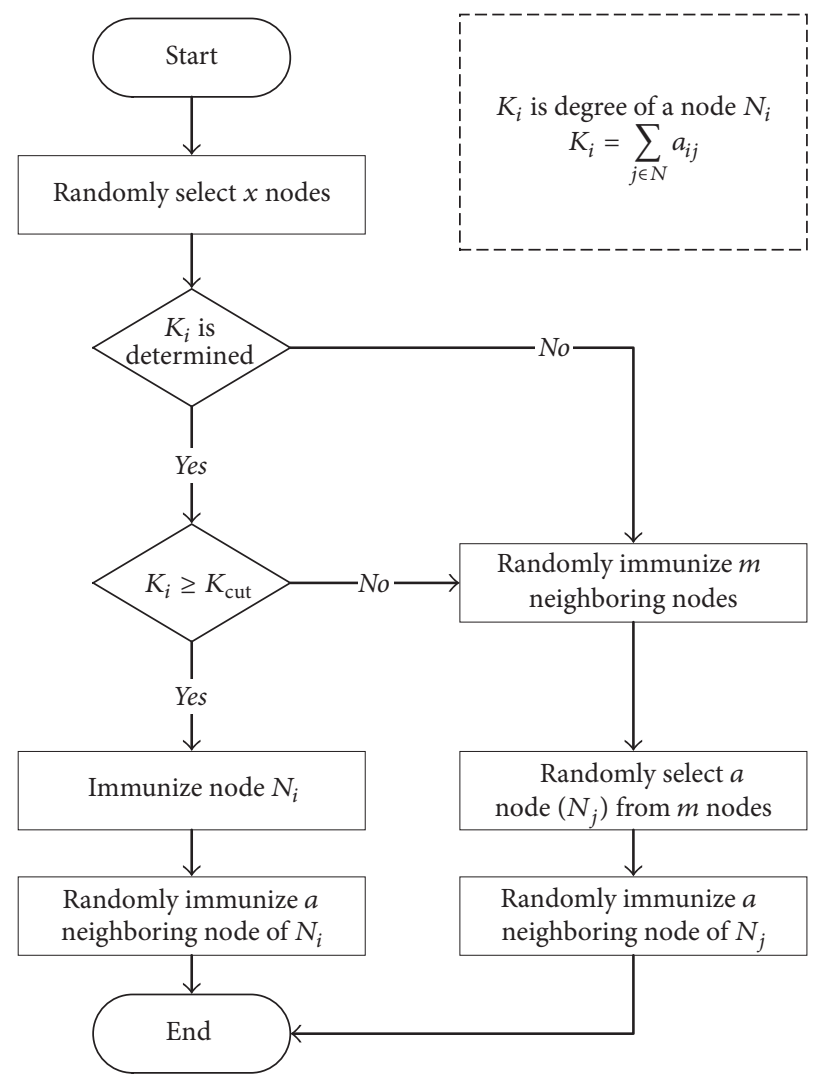

FIgURE 4: The improved acquaintance immunization strategy.

nodes in the network are relatively low, but there are a small number of relatively high degrees of Hub nodes which are the knowledge nodes that most closely related to the design intention.

5.2.2. Identification of Knowledge Groups. In this paper, the cluster coefficient in the network topology is used as the object of analysis to identify and optimize knowledge clusters of the KN-model. The clustering coefficient can be used to describe the ratio of the adjacent nodes to the neighborhood of the nodes in the network, that is, the perfection of the small group structure. The cluster coefficients of the knowledge nodes in the above KN-MODEL can be defined as

$$
\mathrm{CC}_{i}=\frac{3 N_{\Delta}(i)}{N_{3}(i)}
$$




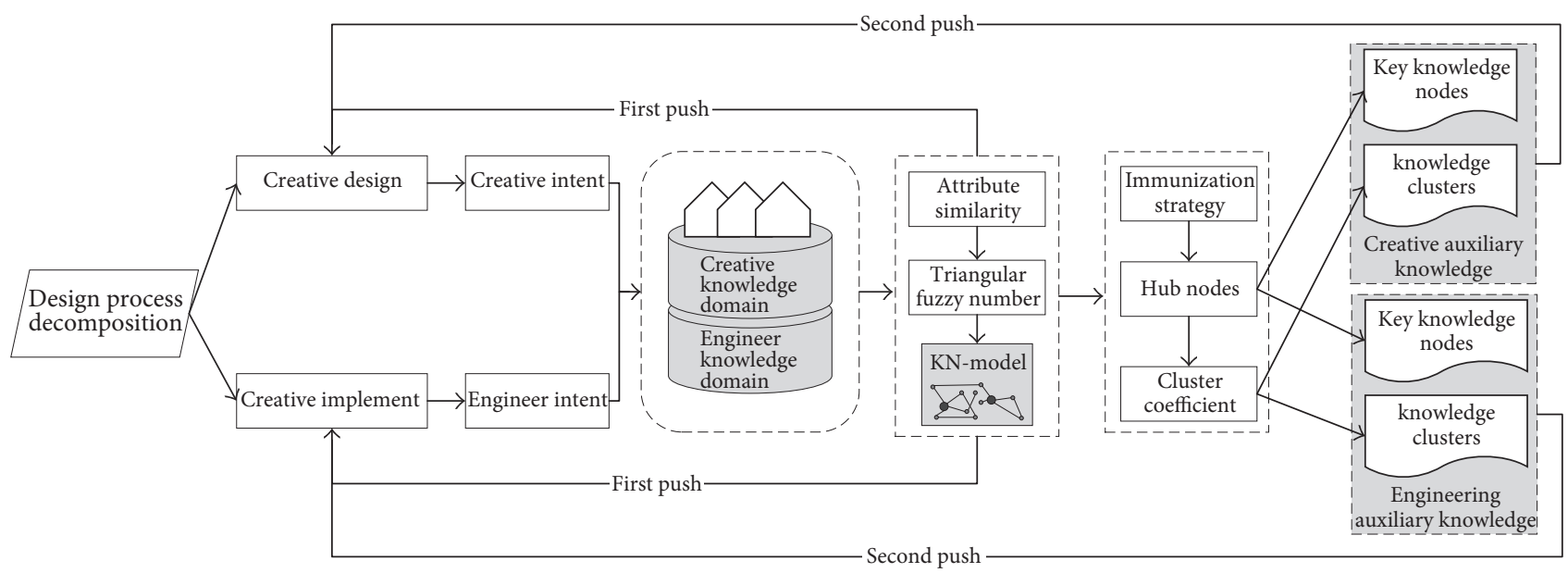

FIGURE 5: The double push strategy of knowledge for product design.

where $N_{\Delta}(i)$ is the total number of triangles containing the node $N_{i}$ in the network

$$
N_{\Delta}(i)=\sum_{k>j} a_{i j} a_{j k} a_{i k}
$$

where $N_{3}(i)$ is the total number of three tuples in the network that contain the node $N_{i}$

$$
N_{3}(i)=\sum_{k>j} a_{i j} a_{i k}
$$

Obviously, $0 \leq \mathrm{CC}_{i} \leq 1$, and $\mathrm{CC}_{i}=0$ if and only if all neighbors are unconnected for any node in the network and $\mathrm{CC}_{i}=1$ if and only if all nodes are connected to each other.

By setting a specific threshold, we can identify the nodes and their small group structure above the threshold, thus mining independent knowledge groups and providing visual design auxiliary information for the subsequent product design.

5.3. Double Push Strategy of Knowledge for Product Design. In order to achieve the goal of assisted innovation, this paper proposes a double push strategy of knowledge for product design (as seen in Figure 5), and the knowledge push process is as follows.

Step 1. The product design process is decomposed into two stages: creative design process and creative implement process, and the creative intention and engineering intention are fully excavated. And then, the product design knowledge base is constructed which includes creative knowledge domain and engineering knowledge domain.

Step 2 (the first push). Through the attribute similarity and triangular fuzzy number proposed in Section 4.1, the knowledge nodes with the maximum weight value are identified, respectively. Then, the directional knowledge push for the first time can be in progress for the creative design process and creative implementation process.
Step 3. Through the acquaintance immune strategy proposed in Section 5.1, some nodes in $\mathrm{KN}$-model are immune, so as to guarantee the novelty of the product design knowledge in the subsequent push link.

Step 4 (the second push). Through the calculation method of cluster coefficient in the network topology proposed in Section 5.2, the knowledge nodes and the knowledge clusters related to creative intention and engineering intention are identified, respectively, then, the supplementary push for the second time can be in progress for the creative design process and creative implementation process.

Through double push strategy of knowledge for product design, the design knowledge can be pushed accurately and directionally, and the aided innovation of design knowledge can be realized.

\section{Implementation and Examples}

The design process of antalgic pump is taken as an illustrative example to demonstrate the double push strategy of knowledge. The analgesic pump is a kind of digital and intelligent medical instrument, which is mainly used to achieve high precision infusion of liquid medicine in clinic through the intelligent make-up device such as motor drive and multichannel data acquisition. Therefore, the design of the analgesic pump belongs to a typical complex design problem.

On the basis of detailed research and analysis of existing products, target users, and usage environment, the design is targeted at lightweight, and the original visual sense of medical equipment should be weakened so as to provide visual and psychological pleasure to the patients. Finally, the creative intention of the product design process is as follows: lively and elegant; the engineering intention is precise control, ease of use, stability, and maintainability. 
In this paper, the engineering intention "lightweight" is taken as an example to verify the effectiveness of the proposed push strategy; other design intents can be pushed in the same way.

6.1. Design Knowledge Modeling. Suppose there are 21 independent design knowledge items in the knowledge domain corresponding to engineering intent "lightweight." Using the method proposed in Section 3.2, 21 independent design knowledge items can be expressed in six-tuple modeling as follows:

$$
\mathrm{KE}=\left(\mathrm{KE}_{\mathrm{id}}, \mathrm{KE}_{t}, \mathrm{KE}_{a}, \mathrm{KE}_{f}, \mathrm{KE}_{i}, \mathrm{KE}_{e}\right)
$$

The meaning of each symbol is as follows: KE: knowledge in the engineering knowledge domain; $\mathrm{KE}_{\mathrm{id}}$ : identification of knowledge; $\mathrm{KE}_{t}$ : the type of knowledge; $\mathrm{KE}_{a}$ : application phase of knowledge; $\mathrm{KE}_{f}$ : the field of knowledge, including material, processing technology, and structure; $\mathrm{KE}_{i}$ : significance degree of knowledge, that is, the importance of knowledge in its domain; $\mathrm{KE}_{e}$ : the expression of knowledge, including data, drawing, manual, and model. The structured description of every design knowledge is shown in Table 1.

6.2. Fuzzy Importance Calculation and First Knowledge Push. There are 3 experts in the decision-making team. For knowledge $K_{1}, K_{2}, \ldots, K_{21}$, the indirect dominance between each element is compared according to its influence on engineering intent "lightweight," then the evaluation matrix can be constructed as follows:

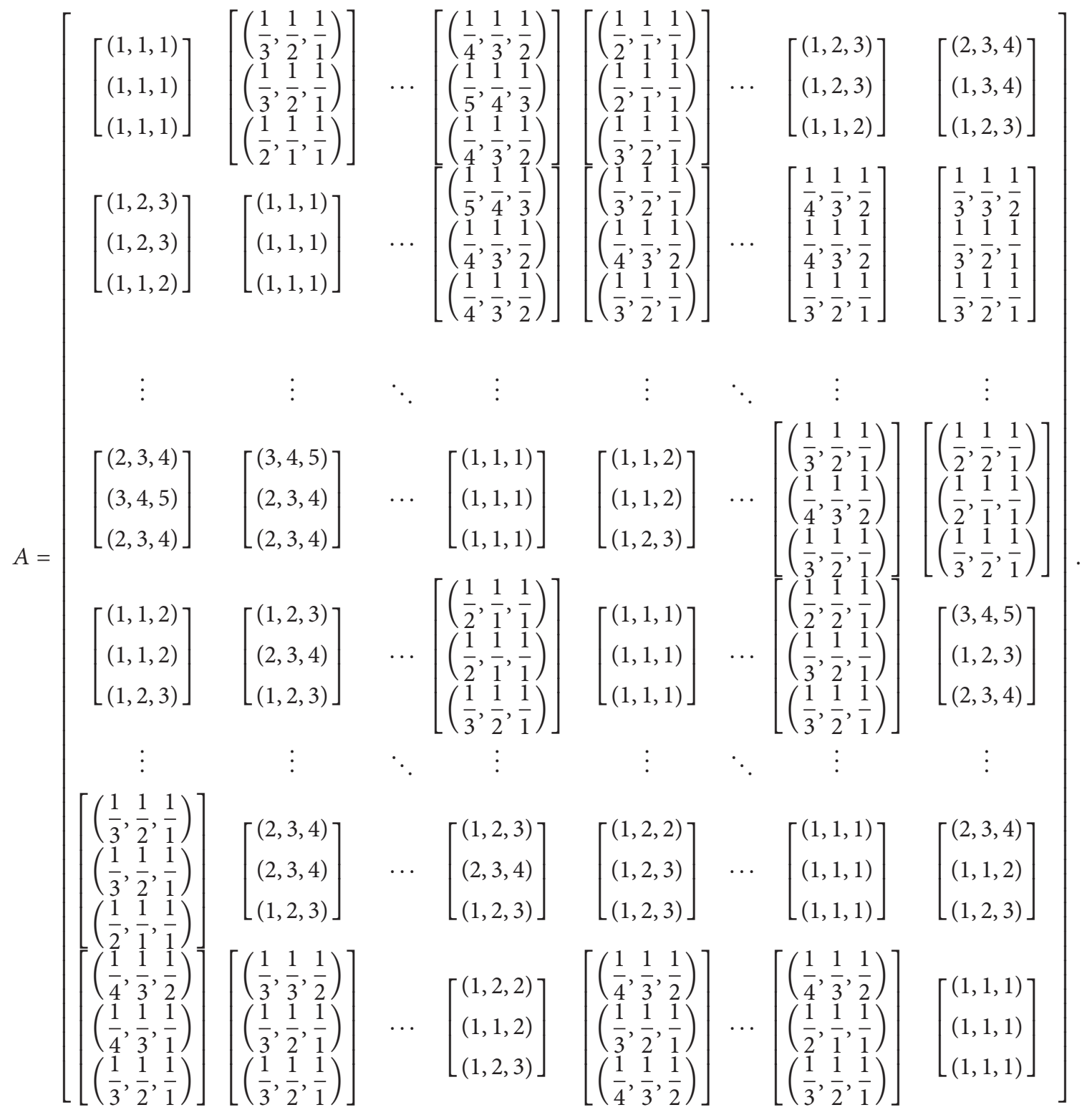




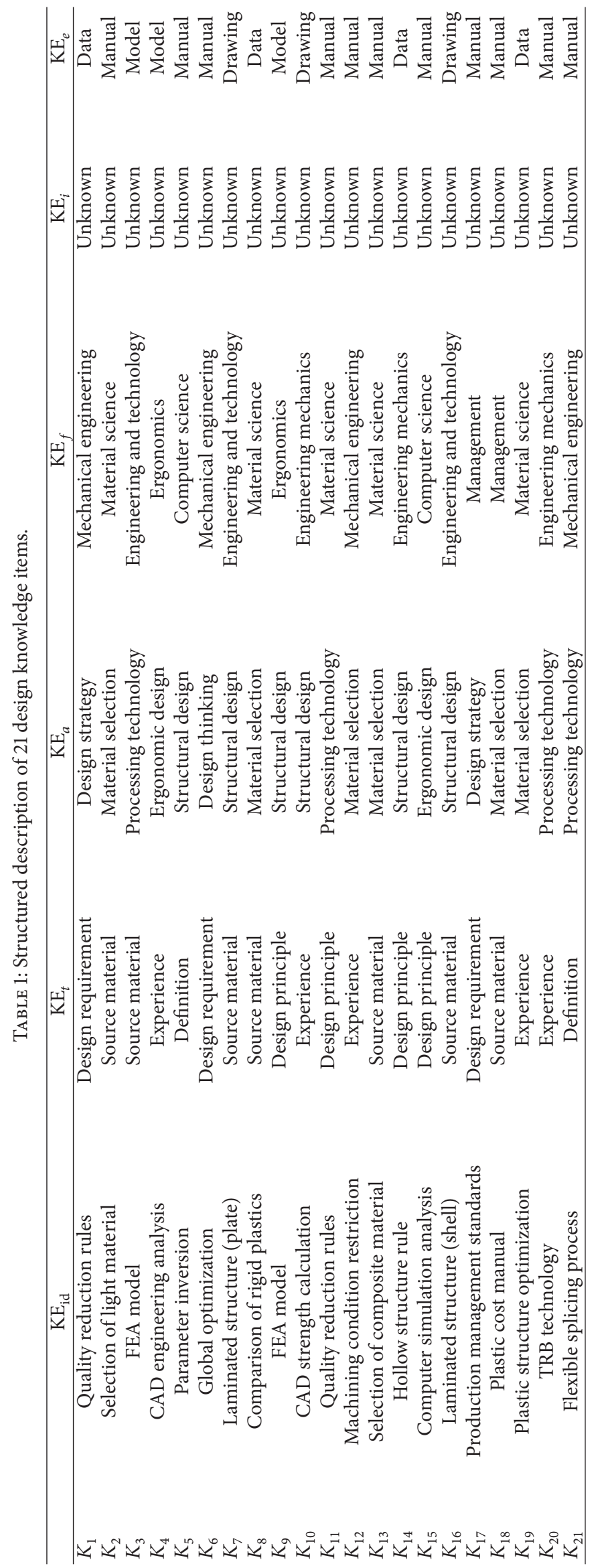


TABLE 2: Statistical results of knowledge nodes retrieved.

\begin{tabular}{ccccccccccccccccccccccc}
\hline & $K_{1}$ & $K_{2}$ & $K_{3}$ & $K_{4}$ & $K_{5}$ & $K_{6}$ & $K_{7}$ & $K_{8}$ & $K_{9}$ & $K_{10}$ & $K_{11}$ & $K_{12}$ & $K_{13}$ & $K_{14}$ & $K_{15}$ & $K_{16}$ & $K_{17}$ & $K_{18}$ & $K_{19}$ & $K_{20}$ & $K_{21}$ & Total \\
\hline$n$ & 8 & 11 & 10 & 7 & 17 & 22 & 16 & 13 & 6 & 8 & 20 & 1 & 9 & 8 & 14 & 12 & 7 & 8 & 3 & 9 & 6 & 215 \\
\hline
\end{tabular}

According to (8), (9), (10), and (12), the evaluation matrix can be transformed as

$A$

$$
=\left[\begin{array}{cccccccc}
{[1,1,1]} & {[0.39,0.67,1.00]} & \cdots & {[0.23,0.31,0.44]} & {[0.44,0.83,1.00]} & \cdots & {[1.0,1.67,2.67]} & {[1.33,2.67,3.67]} \\
{[1.0,1.67,2.67]} & {[1,1,1]} & \cdots & {[0.23,0.31,0.44]} & {[0.31,0.44,0.83]} & \cdots & {[0.28,0.39,0.67]} & {[0.33,0.44,0.83]} \\
\vdots & \vdots & \ddots & \vdots & \vdots & \ddots & \vdots & \vdots \\
{[2.33,3.33,4.33]} & {[2.33,3.33,4.33]} & \cdots & {[1,1,1]} & {[1.00,1.33,2.33]} & \cdots & {[0.31,0.44,0.83]} & {[0.44,0.67,1.00]} \\
{[1.00,1.33,2.33]} & {[1.33,2.33,3.33]} & \cdots & {[0.44,0.83,1.00]} & {[1,1,1]} & \cdots & {[0.39,0.67,1.00]} & {[2.00,3.00,4.00]} \\
\vdots & \vdots & \ddots & \vdots & \vdots & \ddots & \vdots & \vdots \\
{[0.39,0.67,1.00]} & {[1.67,2.67,3.67]} & \cdots & {[0.33,2.33,3.33]} & {[1.00,2.00,2.67]} & \cdots & {[1,1,1]} & {[1.33,2.00,3.00]} \\
{[0.28,0.39,0.83]} & {[0.33,0.44,0.83]} & \cdots & {[1.00,1.67,2.33]} & {[0.28,0.39,0.67]} & \cdots & {[0.36,0.61,0.83]} & {[1,1,1]}
\end{array}\right] .
$$

According to (13)-(16), the initial weight of each knowledge node can be got as

$R$

$=(0.238,0.302,0.314,0.210,0.372,0.392,0.380,0.400,0.174,0.448,0.546,0.114,0.194,0.190,0.222,0.198,0.203,0.135,0.156,0.323,0.308)$.

According to the background data of platform, the frequencies of knowledge nodes retrieved within 48 hours are extracted, and the statistical results are shown in Table 2. We introduce the retrieval factor $\gamma=0.5$ to transform the separation measures into crisp value by using formulas (17) and (18). Synthetical importance of design knowledge $\mathrm{KE}_{i}$ can be calculated (Table 3 ). Therefore, we can rank all knowledge nodes according to their values of $\mathrm{KE}_{i}$.

By setting threshold $\mathrm{KE}_{0}=0.24$, we can identify the most important knowledge nodes $\mathrm{KE}_{6}, \mathrm{KE}_{11}$ in the knowledge domain corresponding to engineering intent "lightweight." These knowledge nodes can be pushed into the corresponding subtask module to implement the first directional knowledge push.

6.3. Knowledge Network Model. According to the six-tuple modeling constructed above, the attribute matrixes of knowledge sets can be established:

$$
E=\left(e_{1}, e_{2}, \ldots, e_{n}\right)^{T},
$$

where row vector $e_{i}(i=1,2, \ldots, n)$ stands for the attribute matrix of knowledge node $K_{i}$. The rules for attribute values are as follows.
$\mathrm{KE}_{\mathrm{id}}$ (identification of knowledge) is used as a function of identification, so the unified value is 1 . There are five types of knowledge $\left(\mathrm{KE}_{t}\right)$ : definition, design requirement, design principles, experience, and source material, corresponding to numerical values $\{1,2,3,4,5\}$. The attribute value of application phase $\mathrm{KE}_{a}$ can be determined by the order of design phase, which means that the attribute value of design thinking phase is 1 , the attribute value of design strategy phase is 2 , the attribute value of creative expression phase is 3 , the attribute value of material selection phase is 4 , the attribute value of processing technology phase is 5 , the attribute value of ergonomic design phase is 6 , and the attribute value of structural design phase is 7 . The attribute value of knowledge field $\mathrm{KE}_{f}$ is similar to the attribute value of application phase, but because of the distance between different knowledge fields, the attribute values should correspond to them. For example, "mechanical engineering" and "engineering and technical science basic disciplines" are similar, so the attribute values are 1 and 2, respectively. "Management" is far from "material science," so the attribute values are 15 and 8, respectively. The attribute value of importance $\mathrm{KE}_{i}$ corresponds to its value. In order to prevent the difference between the values of different attributes, the attribute value of importance will be expanded 
TABLE 3: Synthetical importance of design knowledge.

\begin{tabular}{lc}
\hline & Value \\
\hline $\mathrm{KE}_{1}$ & 0.138 \\
$\mathrm{KE}_{2}$ & 0.177 \\
$\mathrm{KE}_{3}$ & 0.180 \\
$\mathrm{KE}_{4}$ & 0.121 \\
$\mathrm{KE}_{5}$ & 0.226 \\
$\mathrm{KE}_{6}$ & 0.247 \\
$\mathrm{KE}_{7}$ & 0.227 \\
$\mathrm{KE}_{8}$ & 0.230 \\
$\mathrm{KE}_{9}$ & 0.101 \\
$\mathrm{KE}_{10}$ & 0.243 \\
$\mathrm{KE}_{11}$ & 0.320 \\
$\mathrm{KE}_{12}$ & 0.059 \\
$\mathrm{KE}_{13}$ & 0.118 \\
$\mathrm{KE}_{14}$ & 0.114 \\
$\mathrm{KE}_{15}$ & 0.144 \\
$\mathrm{KE}_{16}$ & 0.127 \\
$\mathrm{KE}_{17}$ & 0.118 \\
$\mathrm{KE}_{18}$ & 0.086 \\
$\mathrm{KE}_{19}$ & 0.085 \\
$\mathrm{KE}_{20}$ & 0.182 \\
$\mathrm{KE}_{21}$ & 0.168 \\
\hline
\end{tabular}

by ten times. The attribute value of knowledge expression $\mathrm{KE}_{e}$ refers to the attribute value of knowledge type.

Therefore, the attribute matrix of 21 knowledge nodes can be established as follows:

$$
E=\left[\begin{array}{cccccc}
1 & 2 & 2 & 1 & 1.38 & 1 \\
1 & 5 & 4 & 8 & 1.77 & 4 \\
1 & 5 & 5 & 2 & 1.80 & 3 \\
1 & 4 & 6 & 5 & 1.21 & 3 \\
1 & 1 & 7 & 12 & 2.26 & 4 \\
1 & 2 & 1 & 1 & 2.47 & 4 \\
1 & 5 & 7 & 2 & 2.27 & 2 \\
1 & 5 & 4 & 8 & 2.30 & 1 \\
1 & 3 & 6 & 5 & 1.01 & 3 \\
1 & 4 & 7 & 3 & 2.43 & 2 \\
1 & 3 & 5 & 8 & 3.20 & 4 \\
1 & 4 & 4 & 1 & 0.59 & 4 \\
1 & 5 & 4 & 8 & 1.18 & 4 \\
1 & 3 & 7 & 3 & 1.14 & 1 \\
1 & 3 & 6 & 12 & 1.14 & 4 \\
1 & 5 & 7 & 2 & 1.27 & 2 \\
1 & 2 & 2 & 15 & 1.18 & 4 \\
1 & 5 & 4 & 15 & 0.86 & 4 \\
1 & 4 & 4 & 8 & 0.85 & 1 \\
1 & 4 & 5 & 3 & 1.82 & 4 \\
1 & 1 & 5 & 1 & 1.68 & 4
\end{array}\right],
$$

where row vector of $E$ stands for the attribute matrix of single knowledge node. As to knowledge node $K_{1}$ and knowledge $K_{2}$, the calculation process of attribute similarity between two knowledge nodes is as follows:

$$
\begin{aligned}
& \operatorname{as}_{12}^{1}=\| x_{1}^{1}-\overline{x^{1}}|-| x_{2}^{1}-\overline{x^{1}}||=|| 1-1|-| 1-1|| \\
& =0 \text {, } \\
& \mathrm{as}_{12}^{2}=|| x_{1}^{2}-\overline{x^{2}}|-| x_{2}^{2}-\overline{x^{2}}|| \\
& =|| 2-3.57|-| 5-3.57||=0.14, \\
& \operatorname{as}_{12}^{3}=|| x_{1}^{3}-\overline{x^{3}}|-| x_{2}^{3}-\overline{x^{3}}|| \\
& =|| 2-4.76|-| 4-4.76||=2, \\
& \operatorname{as}_{12}^{4}=|| x_{1}^{4}-\overline{x^{4}}|-| x_{2}^{4}-\overline{x^{4}}|| \\
& =|| 1-5.81|-| 8-5.81||=2.62, \\
& \operatorname{as}_{12}^{5}=|| x_{1}^{5}-\overline{x^{5}}|-| x_{2}^{5}-\overline{x^{5}}|| \\
& =|| 1.38-1.54|-| 1.77-1.54||=0.07, \\
& \operatorname{as}_{12}^{6}=|| x_{1}^{6}-\overline{x^{6}}|-| x_{2}^{6}-\overline{x^{6}}|| \\
& =|| 1-2.95|-| 4-2.95||=0.9 \text {, }
\end{aligned}
$$

In order to facilitate the calculation, we use MATLAB to program above calculation process of attribute similarity. Similarly, the attribute similarity of knowledge nodes can be compared in pairs. The results are shown in Table 4.

By setting threshold ans $s_{0}=0.5$, if ans $(i, j) \leq \operatorname{ans}_{0}=0.5$, there is a relationship between the two knowledge nodes. Then an edge can be created between them. Therefore, the knowledge network model (Figure 6) can be constructed.

6.4. Immunization Strategy in Complex Network. Using the immunization strategy proposed in Section 5.1, the immune process of 21 knowledge nodes is as follows.

Step 1. Randomly select node $K_{10}$ from all knowledge nodes in $K N$-model, setting threshold $K_{\text {cut }}=3$.

Step 2. According to the information shown in Figure 6, the degree of $K_{10}$ can be defined as 4 .

Step 3. Because the degree of $K_{10}$ exceeds the threshold $K_{\text {cut }}=3$, immunize knowledge node $K_{10}$, then randomly immunize a neighboring node of $K_{10}: K_{7}$.

After the completion of immunization strategy, the knowledge network model is reconstructed, which is shown in Figure 7.

6.5. Network Topology Analysis and Second Knowledge Push. By using the method proposed in Section 5.2, topology 


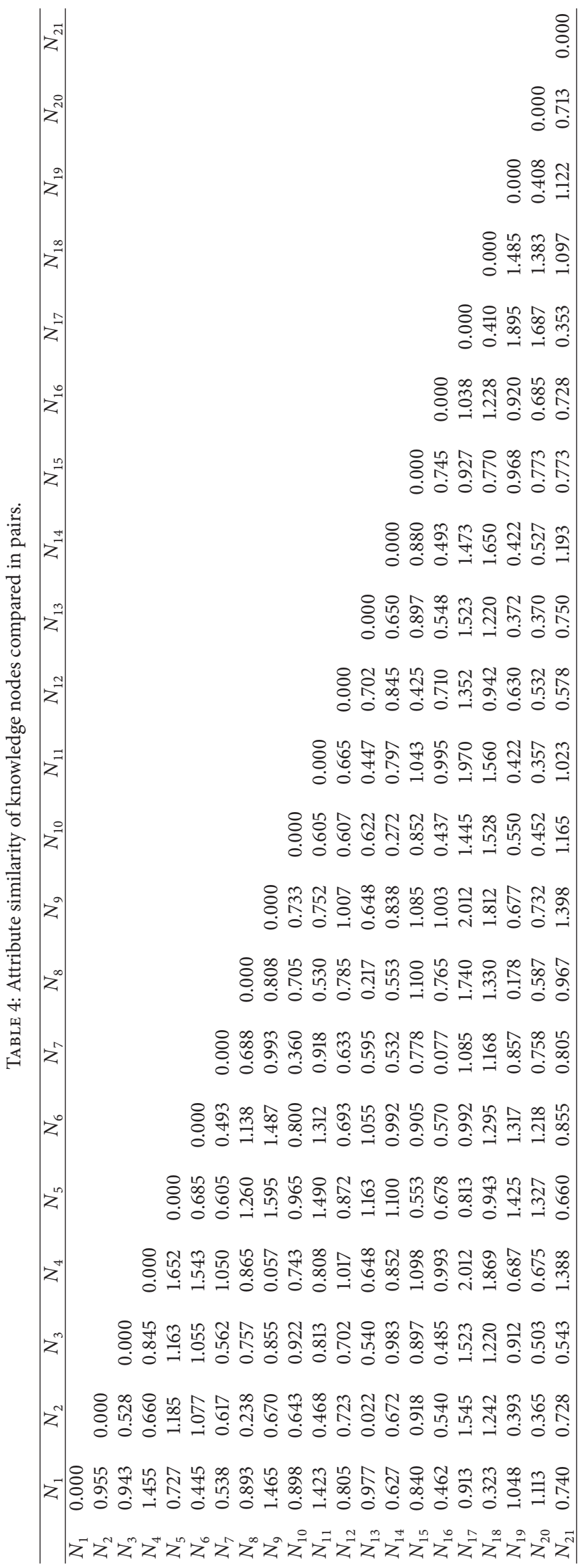


TABLE 5: Result of topology analysis.

\begin{tabular}{cccccccccccccccccccccc}
\hline & $K_{1}$ & $K_{2}$ & $K_{3}$ & $K_{4}$ & $K_{5}$ & $K_{6}$ & $K_{7}$ & $K_{8}$ & $K_{9}$ & $K_{10}$ & $K_{11}$ & $K_{12}$ & $K_{13}$ & $K_{14}$ & $K_{15}$ & $K_{16}$ & $K_{17}$ & $K_{18}$ & $K_{19}$ & $K_{20}$ & $K_{21}$ \\
\hline$K$ & 3 & 5 & 1 & 0 & 0 & 1 & $/$ & 1 & 2 & $/$ & 3 & 1 & 5 & 2 & 1 & 3 & 2 & 2 & 6 & 3 & 1 \\
$\mathrm{CC}$ & 0 & 0.5 & 0 & 0 & 0 & 0 & $/$ & 0 & 1 & $/$ & 1 & 0 & 0.5 & 0 & 0 & 0 & 0 & 0 & 0.4 & 1 & 0 \\
\hline
\end{tabular}

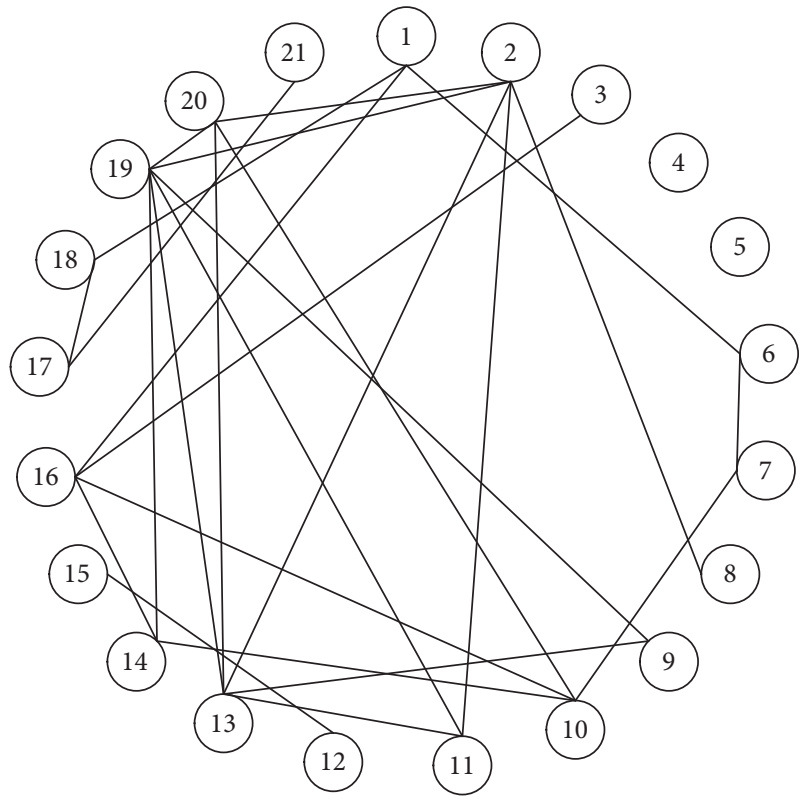

FIGURE 6: The knowledge network model of 21 knowledge nodes.

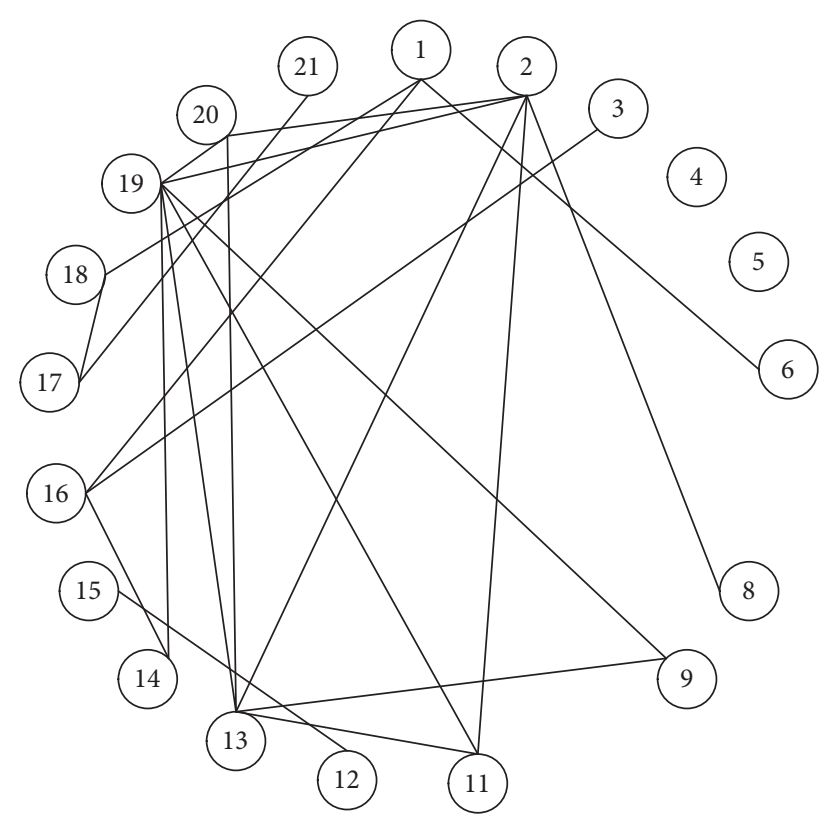

FIgURE 7: The reconstructed knowledge network model.

analysis of the reconstructed knowledge network model (Figure 7) can be carried out. The calculation results of the degree of each knowledge node and clustering coefficients in the network topology are shown in Table 5. Thus, the topology of the reconstructed knowledge network model is plotted, as shown in Figure 8.

The statistical description of the knowledge network model is as follows:

(1) Separate out a Hub node: $K_{19}$.

(2) Separate out a closely related knowledge group: $C_{A}\left\{K_{19}, K_{2}, K_{13}, K_{11}, K_{9}, K_{20}\right\}$.

(3) Separate out two knowledge groups that are not closely related: $C_{B}\left\{K_{14}, K_{16}, K_{3}, K_{1}, K_{6}, K_{18}, K_{17}, K_{21}\right\}$ and $C_{C}\left\{K_{15}, K_{12}\right\}$.

Among them, the knowledge group associated with the Hub node $K_{19}$ is $C_{A}\left\{K_{19}, K_{2}, K_{13}, K_{11}, K_{9}, K_{20}\right\}$. Therefore, Hub node $K_{19}$ and knowledge group $C_{A}\left\{K_{19}, K_{2}, K_{13}\right.$, $K_{11}, K_{9}, K_{20}$ \} can be pushed into the corresponding subtask module to implement the second directional knowledge push.

6.6. Program Implementation. Double push strategy of knowledge for product design proposed in this paper can be implemented with the help of cloud platform "http://www.sunhooyun.com/". Based on ASP.NET technology system and Browser/Server mode and supported by the MySQL database and JAVA language, this paper uses the research results to build a design knowledge aided innovation service platform for antalgic pump (Figure 9).

Through the program interface for creative knowledge push shown in Figure 9(a), designers could receive double creative knowledge push to stimulate creative inspiration. Subsequently, designers completed conceptual design of antalgic pump. The conceptual sketches have been plotted as shown in Figure 10, and the final conceptual design is shown in Figure 11.

Then, through the program interface for engineering knowledge push shown in Figure 9(b), designers can receive double engineering knowledge push to assist engineering design. The result of final design of antalgic pump is shown in Figure 12.

\section{Conclusions}

The paper proposed a double push strategy of product design knowledge to implement the effective auxiliary function for product design process based on the theory and method of complex network. From the global perspective, the paper proposed a comprehensive method that can apply in almost all the engineering domain to help designers and engineers obtaining the knowledge they need most. Compared to traditional ones, this method is more effective and more operable, and it can improve the quality of innovative design and shorten the product design cycle. 


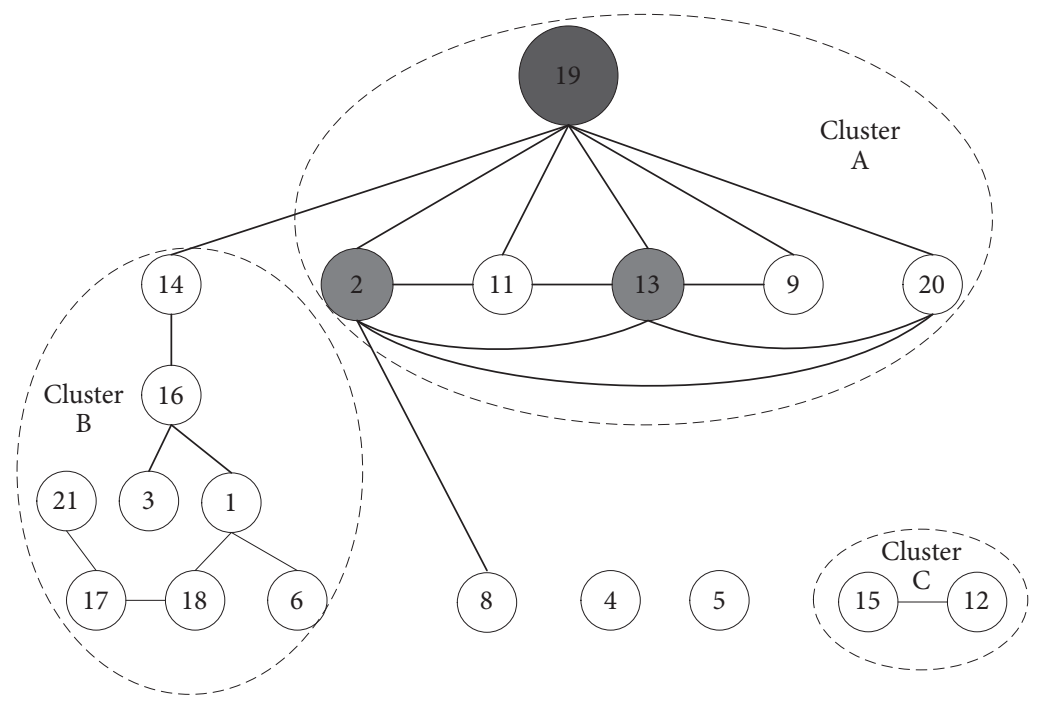

FIGURE 8: The topology of the reconstructed knowledge network model.

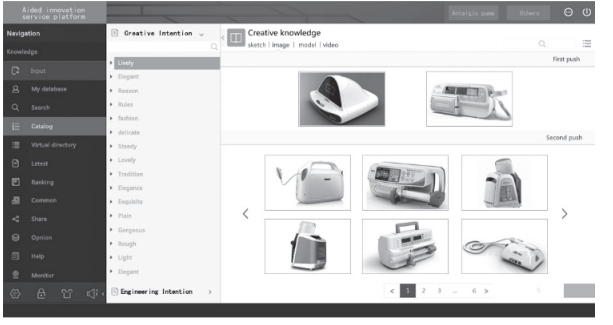

(a) Program interface for creative knowledge push

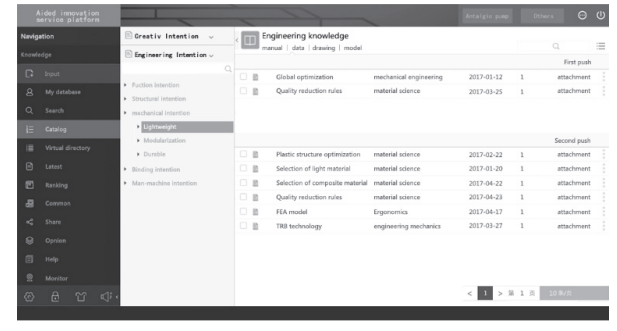

(b) Program interface for engineering knowledge push

FIGURE 9: Design knowledge aided innovation service platform for antalgic pump.
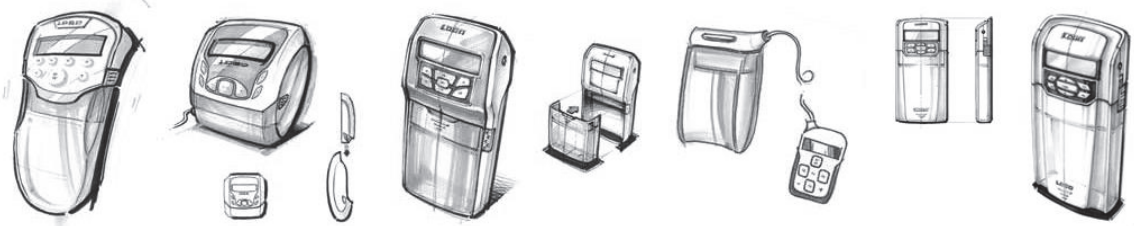

FIGURE 10: Conceptual sketches of antalgic pump.
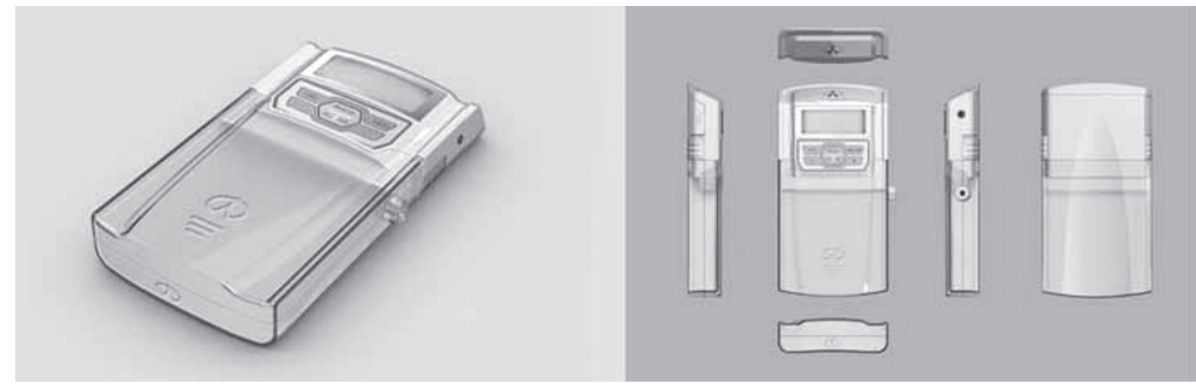

FIGURE 11: Final conceptual design of antalgic pump. 


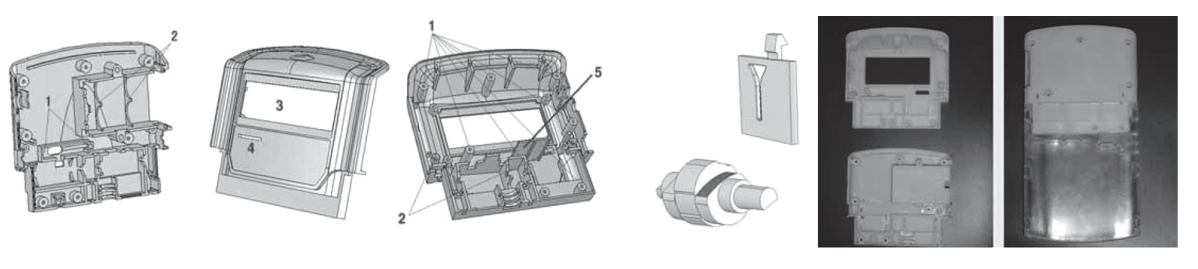

FIGURE 12: Final design of antalgic pump.

\section{Conflicts of Interest}

The authors declare that there are no conflicts of interest regarding the publication of this paper.

\section{Acknowledgments}

This work is supported in part by the National Key Research and Development Program of China (no. 2017YFB1104205) and the National Key Technology R\&D Program of China (no. 2015BAH21F01).

\section{References}

[1] F. Flager and J. J. Haymaker, "Analysis and Optimization Processes in the Building Construction and Aerospace Industries," in Proceedings of the International Conference on Information Technology in Construction, 2007.

[2] D. Chen, J. Ding, M. Gao, D. Ma, and D. Liu, "Form gene clustering method about pan-ethnic-group products based on emotional semantic," Chinese Journal of Mechanical Engineering, vol. 29, no. 6, pp. 1134-1144, 2016.

[3] V. Grover and T. H. Davenport, "General perspectives on knowledge management: Fostering a research agenda," Journal of Management Information Systems, vol. 18, no. 1, pp. 5-21, 2001.

[4] J. Park, "Developing a knowledge management system for storing and using the design knowledge acquired in the process of a user-centered design of the next generation information appliances," Design Studies, vol. 32, no. 5, pp. 482-513, 2011.

[5] G. Jansson, E. Viklund, and H. Lidelöw, "Design management using knowledge innovation and visual planning," Automation in Construction, vol. 72, pp. 330-337, 2016.

[6] N. Matta, G. Ducellier, and C. Djaiz, "Traceability and structuring of cooperative knowledge in design using PLM," Knowledge Management Research and Practice, vol. 11, no. 1, pp. 53-61, 2013.

[7] Y. Feng, S. Zhang, Y. Gao, J. Cheng, and J. Tan, "Intelligent push method of CNC design knowledge based on feature semantic analysis," Jisuanji Jicheng Zhizao Xitong/Computer Integrated Manufacturing Systems, CIMS, vol. 22, no. 1, pp. 189-201, 2016.

[8] Y. Liang, S. Zhang, X. Liu, and C. Wu, "Product design knowledge dynamic push technology based on variable-weight layered spreading activation model," Jisuanji Jicheng Zhizao Xitong/Computer Integrated Manufacturing Systems, CIMS, vol. 21, no. 12, pp. 3107-3118, 2015.

[9] F. Wang, Y. Guo, W. Liao, and B. Wu, "Knowledge Push Technology for Complex Mechatronic Products Design Based on Ontology and Variable Precision Rough Set, Applied Mechanics Materials," Applied Mechanics \& Materials, vol. 799, no. 800, pp. 1107-1112, 2015.

[10] D. J. Watts and S. H. Strogatz, "Collective dynamics of 'smallworld' networks," Nature, vol. 393, no. 6684, pp. 440-442, 1998.
[11] A.-L. Barabási and R. Albert, "Emergence of scaling in random networks," Nature, vol. 286, no. 5439, pp. 509-512, 1999.

[12] A. Yassine and D. Braha, "Complex Concurrent Engineering and the Design Structure Matrix Method," Concurrent Engineering Research and Applications, vol. 11, no. 3, pp. 165-176, 2003.

[13] C. Meier, A. A. Yassine, and T. R. Browning, "Design process sequencing with competent genetic algorithms," Journal of Mechanical Design, vol. 129, no. 6, pp. 566-585, 2007.

[14] D. Braha and Y. Bar-Yam, "The statistical mechanics of complex product development: Empirical and analytical results," Management Science, vol. 53, no. 7, pp. 1127-1145, 2007.

[15] D. Braha and Y. Bar-Yam, "Topology of large-scale engineering problem-solving networks," Physical Review E: Statistical, Nonlinear, and Soft Matter Physics, vol. 69, no. 1, 2004.

[16] P. Fan, G. Qi, and T. Yu, "Network analysis method for commonality analysis of parts modules in modular product platform," Computer Integrated Manufacturing Systems, vol. 19, no. 5, pp. 918-925, 2013.

[17] X. Liu, G. Qi, J. Fu, B. Fan, and J. Xu, "A design process model of integrated morphological matrix and conflict resolving principles," Journal of Zhejiang University (Engineering Science), vol. 46, no. 12, pp. 2243-2251, 2012.

[18] M. Chen, Y. Ji, and G. Qi, "Parameter network modeling and application of design changes for ETO product," Manufacturing Automation, vol. 32, no. 2, pp. 10-14, 2010.

[19] K. Yang, Y. Li, Y. Xiong, W. Li, and H. Na, "Knowledge driven product innovation design based on complex network," Computer Integrated Manufacturing Systems, vol. 21, no. 9, pp. 22572269, 2015.

[20] X. Li, S. Yu, Q. Wang, and C. Wen, "A method for emotional model construction oriented to industrial designer sketch recognition," in Proceedings of the 8th International Conference on Intelligent Human-Machine Systems and Cybernetics, IHMSC 2016, pp. 480-483, China, September 2016.

[21] W.-J. Wang, S.-H. Yu, S.-X. Wang, J. Chen, and L. Deng, "Multilevel comprehensive evaluation and decision making of ergonomics," Discrete Dynamics in Nature and Society, vol. 2015, Article ID 689203, 2015.

[22] R. Cohen, S. Havlin, and D. Ben-Avraham, "Efficient immunization strategies for computer networks and populations," Physical Review Letters, vol. 91, no. 24, Article ID 247901, 2003. 


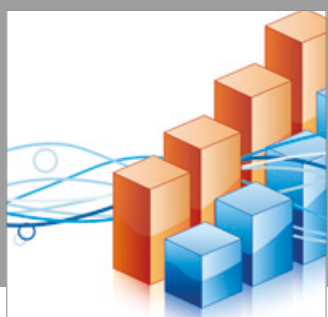

Advances in

Operations Research

vatersals

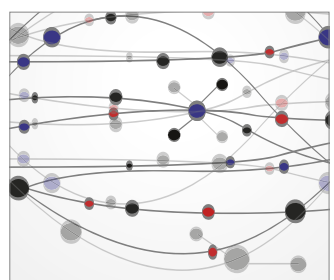

\section{The Scientific} World Journal
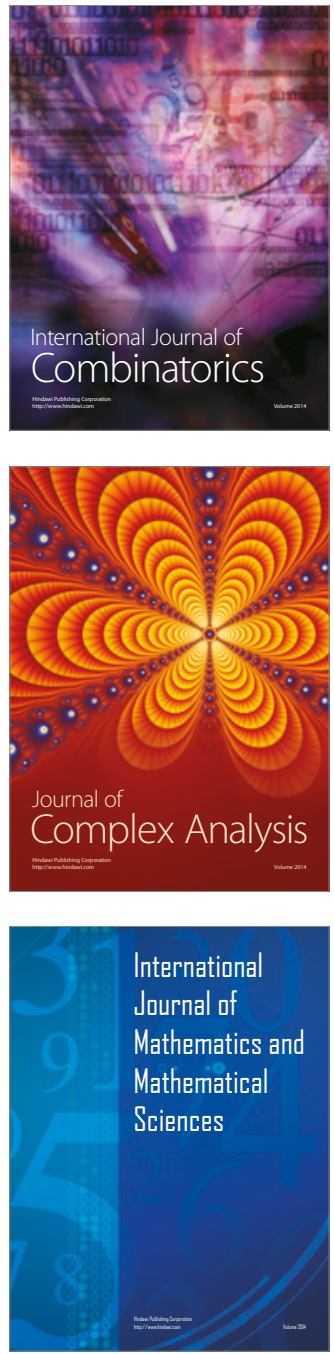
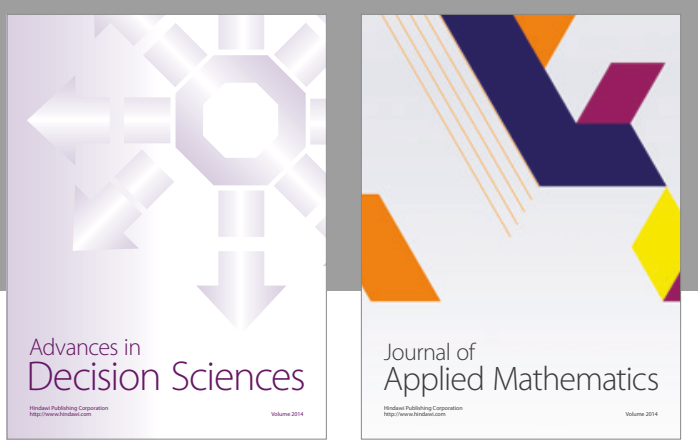

Algebra

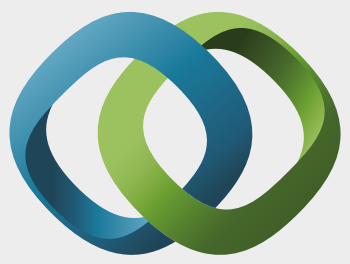

\section{Hindawi}

Submit your manuscripts at

https://www.hindawi.com
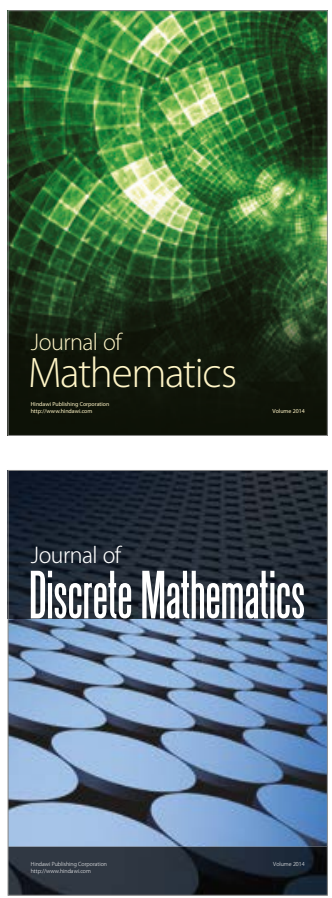

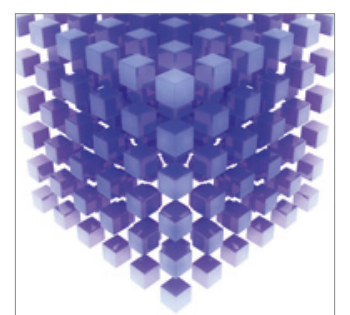

Mathematical Problems in Engineering
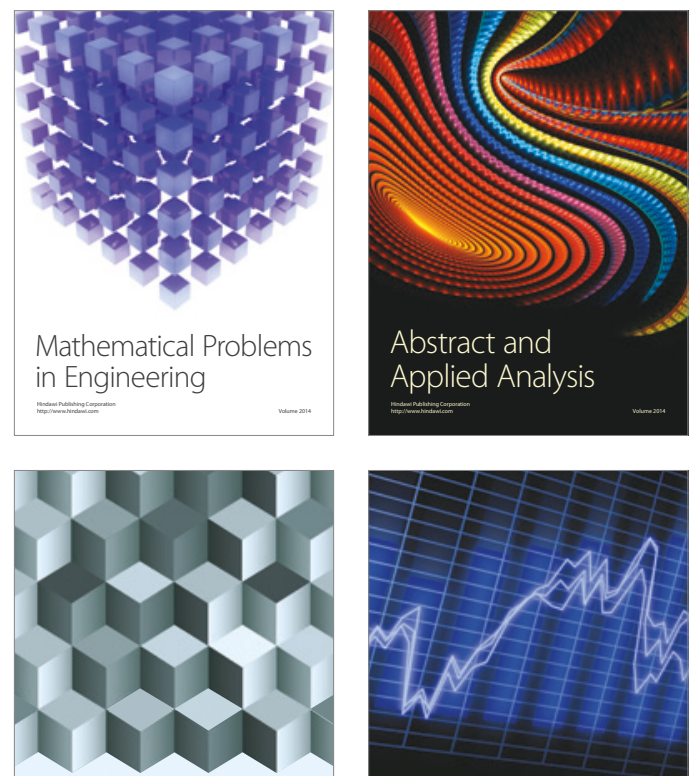

Journal of

Function Spaces

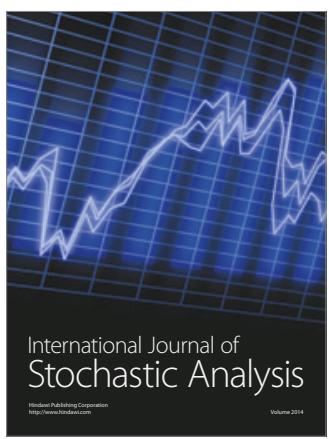

Probability and Statistics
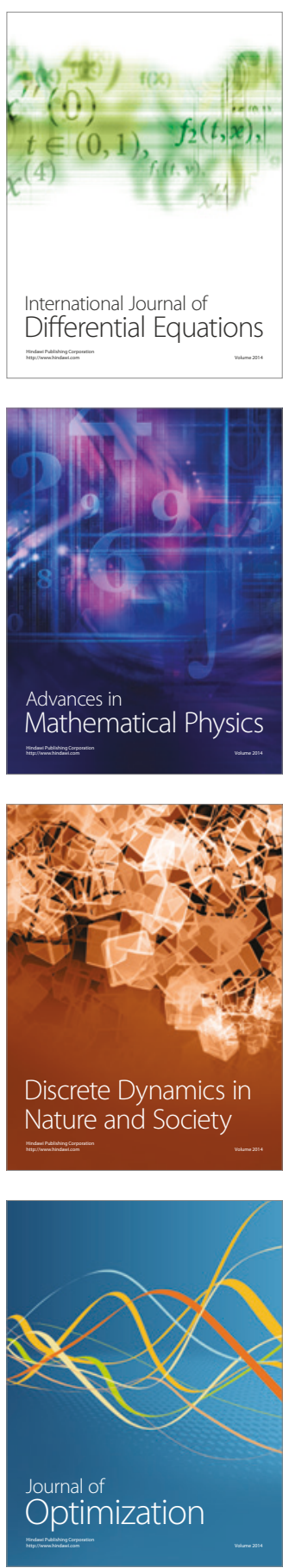